\title{
Características clínicas de 99 pacientes hospitalizados con neumonía por infección de Coronavirus 2019, desde el 15 de marzo hasta el 31 de mayo del 2020
}

\author{
Roberto Proaño A. [1] \\ 1. Médico Especialista en Medicina Interna. Ecuador. \\ Doi: https://doi.org/10.23936/pfr.v5i3.182 \\ PRÁCTICA FAMILIAR RURAL | Vol.5 | No.3 | Noviembre 2020 | Recibido: 25/05/2020 | Aprobado:
}

Cómo citar este artículo

Proaño, R. Características clínicas de 99 pacientes hospitalizados con neumonía por infección de Coronavirus 2019, desde el 15 de marzo hasta el 31 de mayo del 2020. Práctica Familiar Rural. 2020 noviembre; 5(3).

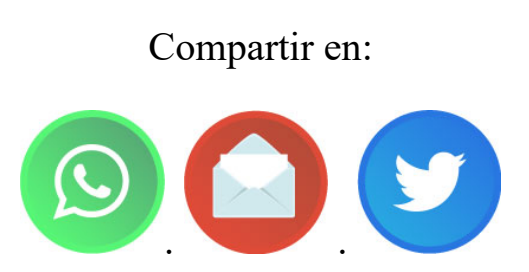

\section{Resumen}

Introducción: A finales del 2019, un nuevo coronavirus fue identificado como causa de casos de Neumonía aislada en Wuhan, una ciudad en Hubei provincia de China, esta rápida expansión, alcanzó pronto los niveles de pandemia, afectando a nivel mundial. En febrero 2020 la Organización Mundial de la Salud, designó como COVID-19 al virus que provoca el SARS.CoV-2 (Síndrome respiratorio agudo severo)(1).

Diversos estudios observacionales se han realizado desde inicios de la pandemia, reportando las manifestaciones clínicas que incluyen fiebre, tos no productiva, disnea, mialgia, fatiga, leucopenia, y evidencia radiológica de Neumonía, posteriormente se reportó una tendencia a desarrollar enfermedad grave en los adultos mayores masculinos(2).

Objetivos: El objetivo de esta serie de casos, fue describir las características clínicas de 
los primeros 99 pacientes con Neumonía con infección por COVID-19, y comparar la evolución de los casos de acuerdo al manejo implementado.

Materiales y Métodos: reporte y descripción de una serie de casos

Resultados: Describir las características clínicas de una población urbano rural ecuatoriana con Neumonía por COVID-19.

Conclusión: Se encontró una edad promedio de 58 años, el 51,5\% (51 pacientes ) fueron mujeres, de estos el 13,1\% (13 pacientes) fueron derivados a Terapia Intensiva o requirieron ventilación mecánica, un total de 55,6\% (55) pacientes fueron dados de alta al domicilio, con una mortalidad hospitalaria en general de $17.2 \%$.

Palabras clave: SARS-CoV-2, COVID-19, Factores de riesgo, características clínicas.

\title{
Clinical characteristics of 99 patients hospitalized with pneumonia due to Coronavirus infection 2019, from March 15 to May 31, 2020
}

\begin{abstract}
Introduction: At the end of 2019, a new coronavirus was identified as a cause of cases of isolated pneumonia in Wuhan, a city in Hubei province of China, this rapid expansion soon reached pandemic levels, affecting the world. In February 2020, the World Health Organization designated the virus that causes SARS. CoV-2 (Severe Acute Respiratory Syndrome) (1), as COVID-19.

Various observational studies have been carried out since the beginning of the pandemic, reporting clinical manifestations that include fever, non-productive cough, dyspnea, myalgia, fatigue, leukopenia, and radiological evidence of Pneumonia, later a tendency to develop serious disease in adults was reported older male (2).

Objectives: The objective of this series of cases was to describe the clinical characteristics of the first 99 patients with Pneumonia with COVID-19 infection, and to compare the evolution of the cases according to the management implemented.

Materials and Methods: report and description of a series of cases

Results: Describe the clinical characteristics of an urban rural Ecuadorian population with COVID-19 Pneumonia.

Conclusion: An average age of 58 years was found, 51.5\% (51 patients) were women, of these $13.1 \%$ (13 patients) were referred to Intensive Care or required mechanical ventilation, a total of $55.6 \%$ (55) patients were discharged home, with an overall hospital mortality of $17.2 \%$.
\end{abstract}

Keywords: SARS-CoV-2, COVID-19, Risk factors, clinical characteristics

\section{Introducción}

A finales del 2019, un nuevo coronavirus fue identificado como causa de casos de Neumonía aislada en Wuhan, una ciudad en Hubei provincia de China, esta rápida expansión, alcanzó pronto los niveles de pandemia, afectando a nivel mundial (1).

En febrero 2020 la Organización Mundial de la Salud, designó como COVID-19, al Coronavirus de tipo 2 causante del Síndrome Respiratorio Agudo Severo (SRAS-CoV-2).

Diversos estudios observacionales se han realizado desde inicios de la pandemia, reportando las manifestaciones clínicas que incluyen fiebre, tos no productiva, disnea, mialgia, fatiga, leucopenia, y evidencia radiológica de Neumonía, posteriormente se reportó una tendencia a desarrollar enfermedad grave en los adultos mayores masculinos (2) 
Este estudio se enfoca en describir las características clínicas, epidemiológicas, laboratorio, características radiológicas, tratamiento y resultados de los primeros pacientes que fueron hospitalizados con infección por Coronavirus (COVID-19), desde el 15 de Marzo hasta el 31 de Mayo del 2020, todos los pacientes ingresados al Hospital Básico de Machachi, con diagnóstico de Neumonía atípica, en este periodo, fueron enrolados.

Se aspira que los hallazgos de este estudio puedan informar a la comunidad sobre la emergencia de este nuevo coronavirus y sus características clínicas en la población ecuatoriana.

\section{Reacción en Cadena de la Polimerasa Transcripción Reversa (PCR-RT) para nCOV.}

Las muestras de hisopados nasofaringeos fueron recolectadas de los pacientes con sospecha de infección de COVID-19, luego de la recolección de la muestra, fue colocada en tubos con $150 \mathrm{uL}$ de solución para preservación del virus, de 2 a 8 grados centígrados hasta el inmediato transporte a los laboratorios del INSPI (Instituto Nacional de Investigación en Salud Pública), ya en el mismo proceden según protocolo del procesamiento de muestra, utilizando gradientes de temperatura para la amplificación de un segmento de $\mathrm{ADN}$, usando ciertos reactivos como los iniciadores o primers, la enzima polimerasa, el buffer de la enzima, $\mathrm{MgCl} 2$, dNTPs, ADN molde, y agua. Este método tiene 3 etapas que forman un ciclo, el cual se repite, usual mente la PCR posee entre 35 a 45 ciclos. La primera es la etapa de denaturación, en ella las hebras de ADN se separan debido a que se rompen los puentes de hidrogeno, por las altas temperaturas a la que es sometida la reacción (94-95

${ }^{\circ} \mathrm{C}$ ). La segunda etapa es la hibridación, en la cual el "primer" o iniciador se une al ADN molde, esta etapa es crucial, puesto que, si el primer no se ancla, no se producirá la amplificación pudiendo dar resultados falsos negativos, para esto es necesario encontrar la temperatura de hibridación correcta $45-65^{\circ} \mathrm{C}$. La tercera etapa se conoce como la extensión, aquí la polimerasa sintetizará una nueva hebra de ADN a partir del primer anclado, esta fase se produce a $72^{\circ} \mathrm{C}$.

\section{Materiales y métodos}

\section{Diseño del estudio y participantes}

Esta serie de casos fue aprobada por el comité de ética del Hospital Básico de Machachi MSP (Ministerio de Salud Pública del Ecuador), todos los pacientes ingresados al Hospital Básico de Machachi, con diagnóstico de Neumonía atípica desde el 15 de Marzo hasta el 31 de Mayo del 2020, fueron ingresados al estudio, se obtuvo el consentimiento verbal de cada paciente. El Hospital Básico de Machachi, localizado en la Provincia de Pichincha, Cantón Mejía, generó la necesidad de contar con una sala de atención para pacientes con COVID-19 durante la pandemia, los pacientes en este estudio fueron diagnosticados según los lineamientos de las guías interinas de la Organización Mundial de la Salud (OMS) (3).

Los resultados clínicos, como las altas, las transferencias, mortalidad, estancia hospitalaria fueron monitorizadas desde marzo hasta junio del 2020

\section{Recolección de datos}

Los datos de los pacientes fueron analizados por el equipo investigador, las características epidemiológicas, clínicas, laboratorio, radiología y resultados de tratamientos fueron obtenidos a partir de la historia clínica.

Los datos fueron revisados por el equipo investigador, la información incluida fue, datos demográficos, historia médica, antecedentes patológicos, historia de exposición, síntomas, signos, 
hallazgos de laboratorio, radiografía estándar de tórax, medidas terapéuticas, (antivirales, corticoides, soporte respiratorio, anticoagulación). El SARS (Síndrome Respiratorio Agudo Severo) fue definido según la recomendación de Berlin4, el tiempo de duración desde la presentación de la enfermedad al ingreso en el hospital, disnea, SARS y el ingreso a UTI (Unidad de Terapia Intensiva) fueron considerados.

\section{Análisis estadístico}

Las variables categóricas fueron descritas con tasa de frecuencias y porcentajes, las variables continuas fueron descritas usando media, mediana, valor de rango intercuartil. La comparación entre las variables continuas fue hecha con la prueba de " $t$ " para grupo independiente con una distribución normal de los datos, cuando no hubo distribución normal de los mismos, se optó por la aplicación de test de Mann-Withney.

Las proporciones de las variables categóricas, fueron comparadas usando la prueba de x2, cuando los datos fueron limitados, se usó el test exacto de Fisher. Todo fue analizado utilizando el SPSS (Stadistical Package for the Social Sciencies) versión 13.0 software (SPSS Inc), se aceptó un error tipo alfa de 0.05 de significancia estadística entre grupos. Dado el potencial para el error tipo I se recomienda que los hallazgos sean interpretados en contexto descriptivo.

\section{Resultados}

En este estudio, se incluyó a los primeros 99 pacientes hospitalizados con diagnóstico confirmado de Neumonía por coronavirus mediante la prueba de PCR RT (Reacción en cadena de la polimerasa; la edad promedio fue de 58 años (RIQ 25-95 años), el 51,5\% (51 pacientes) fueron mujeres, de estos el 13,1\% (13 pacientes) fueron derivados a Terapia Intensiva o requirieron ventilación mecánica, un total de 55,6\% (55) pacientes fueron dados de alta al domicilio, con uso de anticoagulación ambulatoria el $31,3 \%$ (31 pacientes).

El 17,2\% (17 pacientes), fallecieron, la patología concomitante asociada más frecuente fue la Hipertensión arterial $(21,2 \%)$ seguido de la Diabetes tipo $2(12,1 \%)$, el sitio de mayor exposición y de posible contagio fué a nivel domiciliario $(57,7 \%)$ frente a $(42,4 \%)$ en la comunidad; los síntomas más frecuentes que se presentaron en los pacientes fueron, fiebre, disnea, mialgia, fatiga, tos seca, $(91,9 \%, 96 \%, 92,9 \%, 82,8 \%, 77,8 \%)$ respectivamente, los síntomas menos frecuentes reportados en esta serie fueron; dolor abdominal, vómito, dolor de garganta, expectoración, diarrea, anosmiadisgeusia, náusea, cefalea, $(10,1 \%, 13,1 \%, 16,2 \% 23,2 \%, 28,3 \%, 29,3 \%, 69,7 \%)$ respectivamente. La media de duración desde la presentación de los síntomas hasta la búsqueda de asistencia médica hospitalaria, fué de 8 días (RIQ 7-10), teniendo a la fiebre y disnea como principales motivos de consulta.

La frecuencia respiratoria tuvo una media de 29 x minu. (RIQ 25-34 x minu.); la saturación de oxígeno al ingreso en emergencias fue en promedio 79\% al ambiente (RIQ: 75-86\%); los valores de PaFio2 de los pacientes ya ingresados obtuvieron una media de 178 (RIQ 113-210), observando valores tan bajos como hasta de 30; los valores de Fibrinógeno al ingreso, fueron $389 \mathrm{mg} / \mathrm{dl}$ (RIQ 322-456), y luego de 3 días de evolución $344 \mathrm{mg} / \mathrm{dl}$ (RIQ 290-410); el contaje de leucocitos mantuvo una media de 7700 (RIQ 6000-10900) al ingreso en sala COVID, con 6400 (RIQ 49008200 ) al final; los neutrófilos al ingreso una media de 71\% (RIQ 62-79\%) y 62\% (48-74\%) en los días 5 posteriores; los Linfocitos una media de 1530 (RIQ 1197-1870) al ingreso y final 1652 (RIQ 1065-2030); los valores de Dímero D al ingreso presentaron una media de $2418 \mathrm{mg} / \mathrm{dl}$ con mediana en 690 (RIQ 290-1676), con media de $2313 \mathrm{mg} / \mathrm{dl}$ mediana en $620 \mathrm{mg} / \mathrm{dl}$ (RIQ 380-2100) posteriormente; los valores de LDH al ingreso fueron de media $454 \mathrm{mg} / \mathrm{dl}$ (RIQ 341-568), con 370 $\mathrm{mg} / \mathrm{dl}(244-544)$ al final. 
Los pacientes que fallecieron, mantuvieron niveles de Dímero $\mathrm{D}$ en promedio de $4296 \mathrm{mg} / \mathrm{dl}$ $(\mathrm{p}=0,037)$ desde el ingreso y se mantuvo con promedio de $4379 \mathrm{mg} / \mathrm{dl}(0,011)$, al final. Contrastando con la media de $2028 \mathrm{mg} / \mathrm{dl}$ y $1884 \mathrm{mg} / \mathrm{dl}$ al ingreso y alta de los sobrevivientes.

\section{Tabla.1 Características clínicas de pacientes críticos}

\begin{tabular}{|c|c|c|c|c|}
\hline & $\begin{array}{c}\text { NÚMERO } \\
\text { TOTAL }\end{array}$ & $\begin{array}{c}\text { UTI } \\
\text { Ventilación } \\
\text { mecánica }\end{array}$ & $\begin{array}{c}\text { NO UTI } \\
\text { No Ventilación } \\
\text { mecánica }\end{array}$ & $\begin{array}{c}\text { VALOR DE } \\
p\end{array}$ \\
\hline \multicolumn{5}{|l|}{ GÉNERO } \\
\hline MASCULINO & $\begin{array}{l}48(48.5) \\
51(51.5)\end{array}$ & $\begin{array}{l}5(38,5) \\
8(61,5)\end{array}$ & $\begin{array}{l}43(50) \\
43(50)\end{array}$ & \multirow[t]{2}{*}{.43} \\
\hline FEMENINO & & & & \\
\hline $\begin{array}{l}\text { EDAD-MEDIA-RANGO } \\
\text { INTERCUARTIL(RIQ) }\end{array}$ & $58(45-73)$ & $59(26-92)$ & $58(25-95)$ & .13 \\
\hline \multicolumn{5}{|l|}{ SITIO DE EXPOSICIÓN } \\
\hline DOMICILIO & $57(57.6)$ & $8(61.5)$ & $49(57)$ & \multirow[t]{2}{*}{.75} \\
\hline COMUNIDAD & $42(42.4)$ & $5(38.5)$ & $37(43)$ & \\
\hline HIPERTENSIÓN ARTERIAL & $21(21.3)$ & $2(15.4)$ & $19(22.1)$ & .58 \\
\hline DIABETES MELLITUS & $12(12.1)$ & $2(15.4)$ & $10(11.6)$ & .69 \\
\hline FIEBRE & $91(91.9)$ & $13(100)$ & $78(90.7)$ & .25 \\
\hline FATIGA & $82(82.8)$ & $12(92.3)$ & $70(81.4)$ & .33 \\
\hline TOS SECA & $77(77.8)$ & $10(76.9)$ & $67(77.9)$ & .93 \\
\hline ANOREXIA & $39(39.4)$ & $5(38.5)$ & $34(39.5)$ & .94 \\
\hline ESPECTORACION & $23(23,2)$ & $4(30.8)$ & $19(22.1)$ & .49 \\
\hline DISNEA & $92(92.9)$ & $13(100)$ & $79(91.9)$ & .28 \\
\hline DOLOR DE GARGANTA & $16(16.2)$ & $1(7.7)$ & $15(17.4)$ & .37 \\
\hline DIARREA & $28(28.3)$ & $2(15.4)$ & $26(30.2)$ & .26 \\
\hline
\end{tabular}




\begin{tabular}{|c|c|c|c|c|}
\hline NAUSEA & $29(29.3)$ & $4(30.8)$ & $25(29.1)$ & .90 \\
\hline CEFALEA & $69(69.7)$ & $9(69.2)$ & $60(69.8)$ & .96 \\
\hline VOMITO & $13(13.1)$ & $1(7.7)$ & $12(14)$ & .53 \\
\hline DOLOR ABDOMINAL & $10(10.1)$ & $1(7.7)$ & $9(10.5)$ & .75 \\
\hline ANOSMIA/DISGEUSIA & $29(29.3)$ & $5(38.5)$ & $24(27.9)$ & .43 \\
\hline $\begin{array}{l}\text { DÍAS DE PRESENTACIÓN DE } \\
\text { SÍNTOMAS }\end{array}$ & $8(7-10)$ & $9(6-11)$ & $8(5-11)$ & .84 \\
\hline $\begin{array}{l}\text { FRECUENCIA CARDIACA X } \\
\text { MINU }\end{array}$ & $97(85-110)$ & $101(80-122)$ & $96(78-114)$ & .36 \\
\hline $\begin{array}{l}\text { FRECUENCIA RESPIRATORIA } \\
X M I N U .\end{array}$ & $29(25-34)$ & $36(29-43)$ & $29(22-36)$ & .001 \\
\hline $\begin{array}{l}\text { SATURACIÓN DE } \\
\text { INGRESO }\end{array}$ & $79(75-86)$ & $69(58-80)$ & $80(73-87)$ & .000 \\
\hline LEUCOCITOS X 10L & 7.7(6.0-10.9) & $\begin{array}{l}10.16(5.65- \\
14.68)\end{array}$ & $8.95(3.69-14.21$ & .43 \\
\hline LEUCOCITOS X 10L AL FINAL & $6.4(4.9-8.2)$ & $10.33(6.53-14.1)$ & $6.82(3-10.6)$ & .003 \\
\hline NEUTROFILOS \% INGRESO & $71(62-79)$ & $73(67-80)$ & $68(55-80)$ & .16 \\
\hline NEUTROFILOS \% AL FINAL & $62(48-74)$ & $77(70-83)$ & $59(44-73)$ & .037 \\
\hline LINFOCITOS X1OL INGRESO & $1.53(1.19-1.87)$ & $1.78(1.01-2.54)$ & $1.51(0.99-2.11)$ & .18 \\
\hline LINFOCITOS XIOL FINAL & $1.65(1.06-2.03)$ & $1.10(0.40-1.80)$ & $1.72(1.06-2.38)$ & .002 \\
\hline PLAQUETAS X 10L & $316(199-425)$ & $241(85-397)$ & $328(177-479)$ & .058 \\
\hline TP SEGUNDOS & $13.4(11-13)$ & $15.90(8.9-22.8)$ & $13(8.5-17.5)$ & .060 \\
\hline TTP SEGUNDOS & $30(25-32)$ & $30.4(20-40)$ & $30(20-40)$ & .94 \\
\hline
\end{tabular}


LDH U/I INGRESO

LDH U/I ALTA

AST U/L

$A L T U / L$

D-DÍmero mg/L INGRESO

D-DÍmero mg/L AL FINAL

Fibrinógeno mg/dl INGRESO

Fibrinógeno mg/dl AL FINAL

CREATININA $\mathrm{mg} / \mathrm{dl}$

RX OPACIDAD EN VIDRIO

ESMERILADO BILATERAL

PH

$\mathrm{Pa} / \mathrm{FIO} 2 \mathrm{~mm} \mathrm{Hg}$

Lactato $\mathrm{mmol} / \mathrm{L}$

Lesión Renal aguda

INCENTIVOMETRO

ANTIBIOTICOTERAPIA

MORTALIDAD

ALTA DOMICILIO

ANTICOAGULACIÓN

DOMICILIO

$\begin{array}{cccc}454(341-568) & 663(414-912) & 466(271-661) & .002 \\ 370(244-544) & 607(377-837) & 401(169-633) & .009 \\ 47(34-61) & 56(28-85) & 46(29-63) & .065 \\ 51(30-61) & 44(17-61) & 52(22-82) & .35\end{array}$

$2418(290-1676) 7165(890-23234) \quad 1700(848-4248) \quad .004$

$2313(380-2100) 8218(624-22681) \quad 1420(697-3537) \quad .000$ $389(322.456) \quad 426(277-575) \quad 389(272-506) \quad .30$

$344(290-410) \quad 447(259-635) \quad 349(254-444) \quad .09$

$.79(.62-.87) \quad .75(.51-.99) \quad .80(.54-1.06) \quad .56$

$\begin{array}{llll}98(99) & 13(100) & 85(98.8) & .70\end{array}$

7.40(7.38-7.43) $7.38(7.32-7.44) \quad 7.40(7.36-7.44) \quad .090$

$178(113-210) \quad 76(37-116) \quad 178(104-252) \quad .000$

$1.37(1-1.79) \quad 1.7(1.37-2.03) \quad 1.3(0.83-1.77 \quad .003$

$\begin{array}{llll}3(3) & 0(0.0) & 3(3.5) & .49\end{array}$

$\begin{array}{lll}65(65.7) & 0(0.0) & 65(75.6)\end{array}$

$\begin{array}{llll}99(100) & 13(100) & 86(100) & .90\end{array}$

$\begin{array}{llll}17(17.2) & 5(38.5) & 12(14) & .029\end{array}$

$\begin{array}{llll}55(55.6) & 0(0.0) & 55(64) & .000\end{array}$

$\begin{array}{llll}31(31.3) & 0(0.0) & 31(36) & .009\end{array}$ 


\section{Parámetros clínicos y de laboratorio en pacientes críticos y mortalidad.}

Los pacientes que requirieron asistencia en terapia intensiva y ventilación mecánica, $(13,1 \%)$ tuvieron una edad promedio de 59 años frente a los 58 años de los pacientes que no requirieron asistencia ventilatoria mecánica $(\mathrm{p}=0,13)$, la edad en los pacientes fallecidos fue en promedio 71 años, frente a los pacientes que sobrevivieron (82\%) 56 años $(\mathrm{p}=0,00)$. Un $(46,7 \%)$, corresponde a 8 pacientes mujeres, del total de pacientes fallecidos $(17,1 \%)$ frente a $(52,6 \%), 9$ pacientes masculinos $(\mathrm{X} 20,68)$.

Entre los fallecidos se observo a dos pacientes con Diabetes tipo 2 y 6 pacientes con Hipertensión arterial $(11,7 \%$. X2 0,96$),(35,2 \% . X 20,11)$.

En los 17 pacientes fallecidos $(17,1 \%)$ se aplicó protocolo de anticoagulación con HBPM (Heparinas de Bajo Peso Molecular), en dosis de profilaxis o completas según la indicación del caso, se obtuvo (X2 0,64). En cuanto a mortalidad y las características clínicas, se describe, disnea (X2 0,21), fiebre (X2 0,71), fatiga (X2 0,51), tos seca (X2 0,61); de igual manera para necesidad de terapia intensiva y ventilación mecánica y características clínicas; disnea (X2 0,28), fiebre (X2 0,25), fatiga (X2 0,33), tos seca (X2 0,93). Los pacientes fallecidos en la unidad de COVID, tuvieron valores de de $\mathrm{PaFiO} 2$ en una media de $56(\mathrm{p}=0,00)$, valores de $\mathrm{Ph}(\mathrm{p}=0,010)$ por debajo de 7.37, y valores de saturación de oxígeno al ambiente en el momento del ingreso por emergencias en promedio de $69 \%(\mathrm{p}=0,001)$.

Los pacientes que requirieron ventilación mecánica, expresaron valores más altos de Dímero D desde el ingreso a hospitalización $(\mathrm{p}=0,087)$, hasta el final del mismo $(\mathrm{p}=0,00)$, los linfocitos se contaron por debajo de 1000 al ingreso a hospitalización $(\mathrm{p}=0,34)$, mostrando mayor caída previo ingreso a UTI $(0,004)$.

Los pacientes con apoyo de ventilación mecánica y en UTI, registraron frecuencias respiratorias de $>30 \times$ minuto ( $\mathrm{p}=0,000$ ); el nivel de fibrinógeno fue más elevado en estos pacientes $>390 \mathrm{mg} / \mathrm{dl}$, especialmente luego de tres días de evolución hospitalaria $(\mathrm{p}=0,043)$; la saturación de oxígeno se mantuvo por debajo de $80 \%$ especialmente en los pacientes críticos $(\mathrm{p}=0,001)$. Todos los 99 pacientes evaluados presentaron compromiso bilateral de los campos pulmonares, en radiografía convencional de tórax y tomografía simple de tórax.

En los pacientes fallecidos se observó valores de Dímero $\mathrm{D}$ al ingreso $(\mathrm{p}=0,037)$, en el día de fallecimiento $(p=0,011)$, fibrinógeno al ingreso $(p=0,17)$, día de muerte $(p=0,061), \mathrm{LDH}$ al ingreso $(\mathrm{p}=0,003)$, día de fallecimiento $(\mathrm{p}=0,000)$, Linfocitos al ingreso $(0,43)$, linfocitos día de fallecimiento $(0,001)$, los niveles en promedio de Leucocitos al ingreso en los fallecidos fué de 11682, $(\mathrm{p}=0,16)$, en el día de fallecimiento se observó una media de 11958 ( $\mathrm{p}=0,001)$, los neutrófilos media de $75,76 \%$ al ingreso $(p=0,005)$, día de muerte $79,71 \%(p=0,000)$.

\section{Tratamientos y medidas implementadas.}

Muchos de los pacientes recibieron Heparinas de bajo peso molecular, ya sea en profilaxis como a dosis terapéuticas según el protocolo de anticoagulación (99\%), se implemento además la anticoagulación ambulatoria en los pacientes que fueron dados de alta $(31,3 \%)$ y mantenían aún niveles de Dímero D por encima de $1000 \mathrm{mg} / \mathrm{dl}$, todos los pacientes (100\%) recibieron cobertura 
antibiótica con macrólidos (azitromicina) según protocolo de manejo de Covid de HBM, se administró ademas, antibióticos de amplio espectro como Ceftriaxona (34\%), Imipenem (14\%), Vancomicina (8\%) Ampicilina-Sulbactam (67\%), en apenas (4\%) de los pacientes se utilizó Hidroxicloroquina, como parte del protocolo de manejo se implemento el uso de Incentivómetro respiratorio en $(65,7 \%)$ de los pacientes, un total de $(12,6 \%)$ de los pacientes recibieron vasopresores (norepinefrina), un (67\%) de los pacientes con hipoxemia recibió corticoides.

\section{Discusión}

En esta serie de casos de hospitalizados con Neumonía por Coronavirus, se incluyó a los primeros 99 pacientes hospitalizados desde el 15 de Marzo hasta el 31 de Mayo del 2020 con diagnóstico confirmado de Neumonía por coronavirus por PCR RT Hisopado nasal; la edad promedio fue de 58 años (RIQ 25-95 años), el 51,5\% (51 pacientes ) fueron mujeres, de estos el 13,1\% (13 pacientes) fueron derivados a Terapia Intensiva o requirieron ventilación mecánica, un total de 55,6\% (55) pacientes fueron dados de alta al domicilio. El tiempo entre la presentación de los síntomas hasta la búsqueda de asistencia médica hospitalaria, fué de 8 días, los síntomas más frecuentes en la presentación de la enfermedad fueron fiebre, disnea, mialgia, fatiga, tos seca, el sitio de contagio más frecuente fue a nivel domiciliario, todos los pacientes de esta serie presentaron compromiso bilateral en radiografía e imagen tomográfica de tórax. En una buena proporción de pacientes se mantuvo anticoagulación en domicilio, muchos pacientes adultos mayores fallecieron, y todos los pacientes necesitaron algún tipo de apoyo de aporte de oxígeno, desde cánula nasal, hasta ventilación mecánica, en la unidad de COVID del hospital se utilizó además "Venturi", no se dispone de Ventilación mecánica no invasiva, ni de Cánulas de alto flujo.

Se vio un gran incremento del número de pacientes con la infección, desde el inicio de la pandemia en el país (Marzo) en adelante, esto generó la necesidad en los hospitales a nivel nacional a crear unidades para atención exclusiva de COVID 19, una explicación para el aumento del número de contagios tanto en el país como a nivel mundial, es por medio de la estimación del R0, es el número promedio de casos nuevos que genera un caso dado a lo largo de un período infeccioso Esta métrica es útil debido a que ayuda a determinar cuando una enfermedad infecciosa puede dar lugar a un brote epidémico serio, A comienzos de enero, el R0 para el coronavirus era de más de 2 . De acuerdo con estimaciones de estudios chinos (5). Apenas dos meses después los cálculos arrojaron un R0 de casi 6, más del doble de lo previsto al principio. Entender cómo funciona este número es importante para comprender mejor cómo va a desplegarse la enfermedad en la población. Los resultados publicados por el equipo de epidemiología del laboratorio Nacional de los Álamos muestran que el tiempo de duplicación es más bien de 2,3 o 3,3 días, con un R0 de 5.7, esto indica que una persona infectada es capaz de contagiar, de media, a 5,7 personas (6), situando a SARSCOV 2 como uno de los patógenos más contagiosos.

No se observó aumento de la frecuencia de comorbilidades entre los pacientes que entraron a ventilación mecánica o que tuvieron una evolución fatal; ni el género ni la edad fueron factores determinantes para el riesgo de uso de terapia y/o ventilación mecánica, este resultado coincide con anteriores revisiones, donde se demuestra poca asociación con edad, género y necesidad de apoyo ventilatorio mecánico2; con edad se observó diferencia significativa en relación con mortalidad, la edad de los pacientes fallecidos fue en promedio 71 años, frente a los pacientes que sobrevivieron $(82 \%) 56$ años $(\mathrm{p}=0,00)$, una explicación, para esta relación puede ser, que la mayoría de pacientes adultos mayores fallecidos, tenía patologías pulmonares subyacentes sin diagnóstico establecido, lo que provocó una mala evolución con la infección por SARS-COV2. Entre los pacientes fallecidos y los que necesitaron ventilación mecánica, se vio relación con los niveles de $\mathrm{PaFiO} 2$ (56 mmhg), saturación de oxigeno al ingreso $(<69 \%)$, y $\mathrm{Ph}(<7.37)$ de manera significa, esto puede ayudar al clínico a identificar desde el ingreso a emergencias y durante la evolución en hospitalización a los pacientes que pueden tener una mala evolución. 
Las anomalías de laboratorio más frecuentes observadas en este estudio, fueron Dímero D, linfocitos, LDH, Fibrinógeno, leucocitos, neutrófilos, demostrando una relación significativa entre la elevación de los mismos y paciente con evolución crítica, frente a los pacientes que no requirieron cuidados críticos y/o apoyo ventilatorio mecánico. Los valores de biometria que presentaron mayor variabilidad durante la hospitalización, fueron; leucocitos con los que se observó una relación significativa con el riesgo de requerir apoyo ventilatorio (.003), especialmente cuando se dió un incremento de los mismos a partir del 3 día de hospitalización, similar comportamiento se vio con los neutrófilos (.037), el contraje de los linfocitos disminuyeron en los pacientes con presentación crítica de la enfermedad, hubo relación con el ingreso a ventilación mecánica, o cuadro severo, especialmente cuando el contraje de linfocitos disminuía a $<1000$ (.002), esto sugiere que se podría predecir de cierta forma los cuadros clínicos con evolución tórpida o con riesgo de requerir apoyo ventilatorio complejo, los valores de D-dímero se elevaron muy por encima de $>500 \mathrm{mg} / \mathrm{dl}$, con un punto de corte de $1000 \mathrm{mg} / \mathrm{dl}$, a partir del cual se observó una relación significativa con el aumento de riesgo de complicaciones tromboembolicas y mayor mortalidad $(p=.01)$; en varios estudios de coagulopatía y COVID-19, se muestran niveles elevados de D-dímero, en una serie de casos en China con 1099 pacientes, se observó niveles elevados de D-dímero $>0.5 \mathrm{mg} / \mathrm{L}$ (11) con un valor promedio de $2.12 \mathrm{mg} / \mathrm{L}$ en los pacientes no sobrevivientes y valores más bajos $0.61 \mathrm{mg} / \mathrm{L}$ en $\operatorname{los}$ sobrevivientes, se encontró relación significativa con el contraje plaquetario y el tiempo de protrombina (TP), tanto con mortalidad como con curso grave y necesidad de UTI $(\mathrm{p}=.058)$ $(\mathrm{p}=.060)$ respectivamente.

Se tuvo 2 pacientes $(2,1 \%)$ con injuria miocárdica documentada. Estas observaciones coinciden con estudios que reportan relación entre mortalidad y compromiso hematológico (7), la fisiopatología de la infección por SARS-Cov-2, esta relacionada fuertemente con una respuesta inflamatoria agresiva, que da como resultado el daño de las vías aéreas, es así que la severidad de la enfermedad no es solo debido a la infección viral sino también a la respuesta del huésped (8).

Las consecuencias hematológicas de esta nueva infección permitirían a la comunidad medica iniciar nuevos tratamientos, teniendo como metas la anticoagulación y medicamentos antiinflamatorios (9).

Muchos pacientes con COVID-19 severo presentan anomalías de coagulación que pueden simular otras coagulopatías sistémicas asociadas con infecciones severas, como la CID (coagulación intravascular diseminada) o microangiopatía trombótica, pero la COVID-19 tiene otras características (10).

En los pacientes del estudio se utilizó anticoagulación entre dosis profilácticas, hasta dosis completas de hasta $1.5 \mathrm{mg}$ por $\mathrm{Kg}$, sin ningún reporte de sangrados $(0.0 \%)$, lo que sugiere, un gran efecto protrombótico en la COVID-19 con combinación de CID de bajo grado y microangiopatía trombótica pulmonar localizada que tiene impacto sustancial sobre la disfunción de órganos en muchos pacientes severamente afectados $(12,13)$.

La hipoxemia también puede jugar un rol en la trombosis vascular pulmonar (14), otros factores, incluido la ventilación mecánica en pacientes que progresan a SDRA, puede contribuir a este cuadro (15), es así que el estudio se pudo apreciar una media de saturación de oxígeno al ingreso de emergencia de $79 \%$ esto se vio relacionado de manera significativa con mayor riesgo de requerir UTI $(p=.00)$ y mayor riesgo de muerte si esto la saturación se mantiene entre 58 a $80 \%$ hasta el 4 día de hospitalización $(\mathrm{p}=.023)$

Entre los pacientes fallecidos y los que necesitaron ventilación mecánica, hubo relación con los niveles de $\mathrm{PaFiO} 2$ (relación entre la presión arterial de oxígeno y la fracción inspirada de oxígeno (56 mmhg), se vio retraso en decidir intubación de los pacientes, debido en parte a saturación del servicio, y falta de ventiladores, por lo que se vieron pacientes con PaFio2 tan bajas hasta de 30 $\mathrm{mmHg}$, mientras permanecían en espera del uso de ventilación, o transferencia respectiva, la misma que se veía retrasada, por falta de disponibilidad de espacio en las unidades de referencia. 
Durante el período del estudio se reportó un evento de embolia pulmonar con resultado final fatal, dos pacientes con injuria miocárdica con resultado fatal, en ámbas, hubo patología subyacente como Diabetes e Hipertensión arterial, con más de 60 años de edad, en particular se obtuvo valores elevados de troponina cardíaca ( $\mathrm{cTn}$ ), con cambios electrocardiográficos, como parte del manejo en sala COVID, se implementó el uso de electrocariografía al ingreso de los pacientes para registro basal de los mismos, el incremento de los valores de las de troponina cardíaca (cTn), indicador de injuria miocárdica, es común en pacientes con COVID-19 y se asocia con resultados adversos tales como arritmias, y muerte, este incremento es más frecuente en pacientes con condiciones cardiovasculares crónicas y en aquellos con un presentación severa de la COVID-19 (16). La miocarditis, la miocariopatía, la falla cardíaca aguda y la injuria directa por el SARS-CoV-2, son etiologías importantes, pero también las condiciones no cardíacas como el embolismo pulmonar, sepsis, probablemente pueden causar más injuria miocárdica (17), en algunos pacientes críticos una respuesta hiperinflamatoria y la tormenta de citoquinas pueden presentarse, lo que puede contribuir al desarrollo de isquemia miocardica $(18,19)$, de esta manera el uso estructurado de los niveles de cTn en conjunto con electrocardiograma, tienen el potencial de facilitar la estratificación de riesgo, ayudar a tomar decisiones en los pacientes hospitalizados con COVID-19

\section{Antibióticos de amplio espectro}

Durante la hospitalización de los pacientes incluidos en el estudio, se usó antibióticos de amplio espectro, como, Ceftriaxona (34\%), Imipenem (14\%), Vancomicina (8\%) Ampicilina-Sulbactam $(67 \%)$, esto fue considerado, en base a los hallazgos radiológicos y los marcadores inflamatorios compatibles con co-infección bacteriana (20) En estudios observacionales, aproximadamente el 8\% de los pacientes hospitalizados con COVID-19, presentaron una infección bacteriana o fúngica, pero cerca del $72 \%$ fueron tratados con antibióticos de amplio espectro (20).

\section{Antimaláricos}

Más de 200 estudios acerca de cloroquina/ hidroxicloroquina, fármaco que inhibe la entrada viral y la endocitosis del SARS-CoV-2 in vitro y puede tener efecto inmunomulador in vivo pero los datos en pacientes hospitalizados, no demuestran un beneficio claro $(21,22)$, un estudio de cohortes multicéntrico retrospectivo, comparó la mortalidad en hospitalizados, entre pacientes tratados con Hidroxicloroquina plus azitromicina.

Hidroxicloroquina sola, azitromicina sola, y ningún medicamento; no se reportó diferencias entre los grupos (23), en base a la información disponible en el momento, no se incluyó el uso de antimaláricos en el protocolo de manejo de la COVID-19 del hospital, apenas 4 pacientes recibieron hidroxicloroquina debido a que mantenían tratamiento para otras enfermedades concomitantes, el uso de azitromicina se incluyó como parte de la cobertura antibiótica de amplio espectro, y por el aparente efecto inmunomodulador en la COVID-19 a través de los interferones (20).

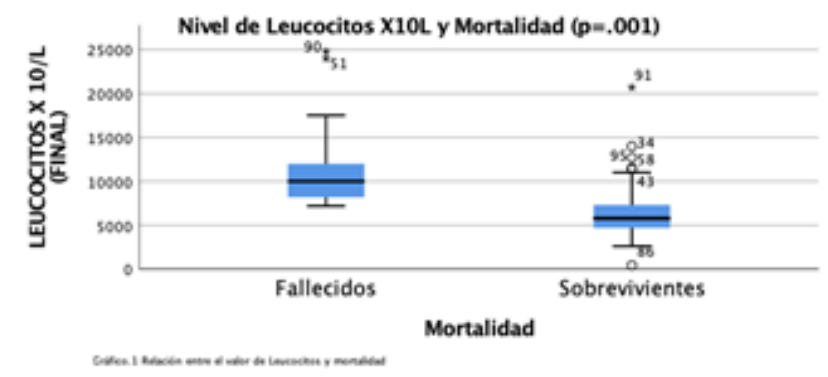



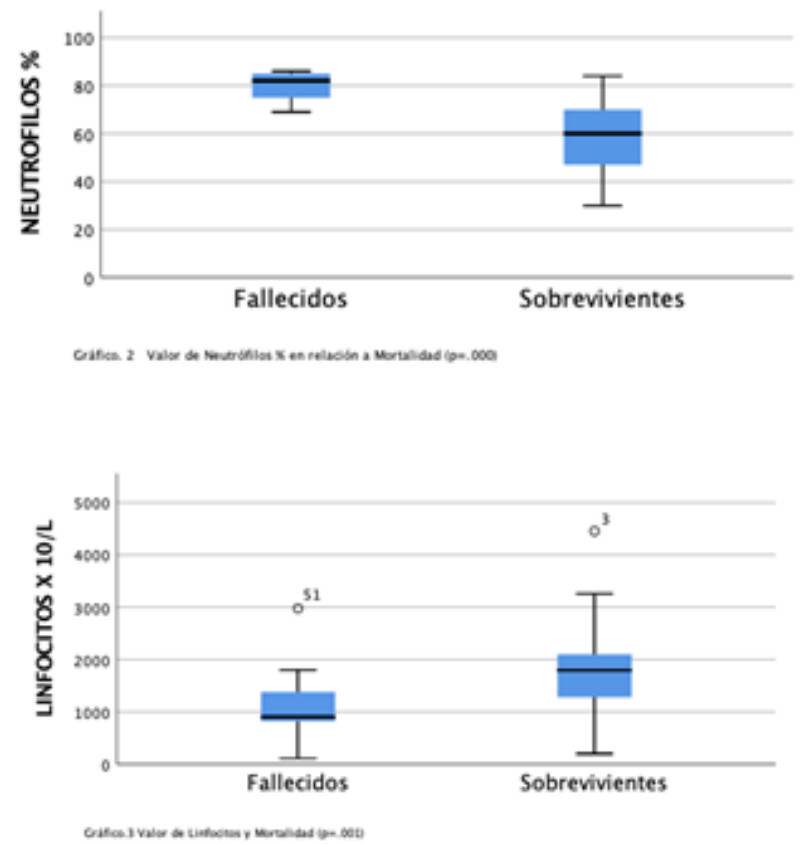

Tabla. 2 características clínicas y mortalidad.

NÚMERO TOTAL FALLECIDO

GÉNERO

MASCULINO

$48(48.5)$

$9(52,9)$

$39(47.6)$

.68

FEMENINO

$51(51.5)$

$8(47,1)$

$43(52.4)$

$58(45-73)$

$71(59-84)$

$56(40-72)$

.00

INTERCUARTIL(RIQ)

SITIO DE EXPOSICIÓN

DOMICILIO

$57(57.6)$

$10(58.8)$

$42(42.4)$

$7(41.2)$

47(57.3)

$35(42.7)$

HIPERTENSIÓN ARTERIAL

21(21.3)

$6(35.3)$

$15(18.3)$

.11

DIABETES MELLITUS

12(12.1)

2(11.8)

$10(12.2)$

.96

FIEBRE

91(91.9)

16(94)

75(91.5)

.71

FATIGA

$82(82.8)$

15(88.2)

67(81.7)

.51

TOS SECA

$77(77.8)$

14(82.4)

63(76.8)

.61 


\begin{tabular}{|c|c|c|c|c|}
\hline ANOREXIA & $39(39.4)$ & $8(347.1)$ & $31(37.8)$ & .47 \\
\hline ESPECTORACION & $23(23,2)$ & $3(17.6)$ & $20(24.4)$ & .54 \\
\hline DISNEA & $92(92.9)$ & $17(100)$ & $75(91.5)$ & .21 \\
\hline DOLOR DE GARGANTA & $16(16.2)$ & $2(11.8)$ & $14(17.1)$ & .58 \\
\hline DIARREA & $28(28.3)$ & $4(23.5)$ & $24(29.3)$ & .63 \\
\hline NAUSEA & $29(29.3)$ & $4(23.5)$ & $25(30.5)$ & .56 \\
\hline CEFALEA & $69(69.7)$ & $12(70.6)$ & $57(69.5)$ & .93 \\
\hline VOMITO & $13(13.1)$ & $0(0)$ & $13(15.9)$ & .07 \\
\hline DOLOR ABDOMINAL & $10(10.1)$ & $0(0)$ & $10(12.2)$ & .12 \\
\hline ANOSMIA/ DISGEUSIA & $29(29.3)$ & $3(17.6)$ & $26(31.7)$ & .24 \\
\hline $\begin{array}{l}\text { DÍAS DE PRESENTACIÓN DE } \\
\text { SÍNTOMAS }\end{array}$ & $8(7-10)$ & $9(6-11)$ & $8(5-11)$ & .52 \\
\hline $\begin{array}{l}\text { FRECUENCIA CARDIACA X } \\
\text { MINU }\end{array}$ & $97(85-110)$ & $98(82-114)$ & $97(78-116)$ & .82 \\
\hline $\begin{array}{l}\text { FRECUENCIA RESPIRATORIA } \\
X M I N U .\end{array}$ & $30(25-34)$ & $34(25-43)$ & $29(22-36)$ & .018 \\
\hline $\begin{array}{l}\text { SATURACIÓN DE } \\
\text { INGRESO }\end{array}$ & $79(75-86)$ & $69(58-80)$ & $81(74-88)$ & .023 \\
\hline LEUCOCITOS X $10 L$ & $9.11(6.0-10.9)$ & $11.68(3.05-20.30)$ & $8.57(4.58-12.56$ & .023 \\
\hline LEUCOCITOS X 10L AL FINAL & $7.28(4.9-8.2)$ & $11.95(6.56-17.35)$ & $6.31(3.50-9.13)$ & .001 \\
\hline NEUTROFILOS \% INGRESO & $69(62-79)$ & $75(67-83)$ & $68(56-79)$ & .005 \\
\hline NEUTROFILOS \% AL FINAL & $61(48-74)$ & $79(72-85)$ & $58(44-71)$ & .000 \\
\hline
\end{tabular}




\begin{tabular}{|c|c|c|c|c|}
\hline LINFOCITOS XIOL INGRESO & $1.58(1.19-1.87)$ & $1.47(.85-2.09)$ & $1.60(1.01-2.18)$ & .40 \\
\hline LINFOCITOS X10L FINAL & $1.65(1.06-2.03)$ & $1.13(0.50-1.76)$ & $1.75(1.09-2.41)$ & .001 \\
\hline PLAQUETAS X 10L & $316(199-425)$ & $259(72-446)$ & $328(183-473)$ & .095 \\
\hline TP SEGUNDOS & $13.4(11-13)$ & $15.98(9.7-22.1)$ & $12(8.3-16)$ & .070 \\
\hline TTP SEGUNDOS & $30(25-32)$ & $32(22-42)$ & $30(20-40)$ & .29 \\
\hline LDH U/I INGRESO & $492(341-568)$ & $689(410-968)$ & $451(280-622)$ & .000 \\
\hline$L D H U / I A L T A$ & $428(244-544)$ & $728(456-1000)$ & $366(184-548)$ & .009 \\
\hline$A S T U / L$ & $47(34-61)$ & $55(33-77)$ & $46(28-64)$ & .13 \\
\hline$A L T U / L$ & $51(30-61)$ & $55(29-81)$ & $50(20-80)$ & .53 \\
\hline D-DÍmero mg/L INGRESO & 2418(290-1676) & $4296(1204-7388)$ & $2028(479-8846)$ & .037 \\
\hline D-DÍmero $m g / L$ AL FINAL & $2313(380-2100)$ & $4379(1710-7048)$ & 1884(441-8180) & .011 \\
\hline Fibrinógeno mg/dl INGRESO & $394(322.456)$ & $422(341-503)$ & $388(260-516)$ & .30 \\
\hline Fibrinógeno mg/dl AL FINAL & $362(290-410)$ & $418(288-548)$ & $351(242-460)$ & .06 \\
\hline CREATININA $\mathrm{mg} / \mathrm{dl}$ & $.79(.62-.87)$ & $.94(.56-.1 .32)$ & $.76(.55-.97)$ & .008 \\
\hline $\begin{array}{l}R X \text { OPACIDAD EN VIDRIO } \\
\text { ESMERILADO BILATERAL }\end{array}$ & $98(99)$ & $17(100)$ & $81(98.8)$ & .64 \\
\hline$P H$ & $7.40(7.38-7.43)$ & 7.37(7.32-7.42) & $7.41(7.37-7.45)$ & .010 \\
\hline $\mathrm{Pa} / \mathrm{FIO} 2 \mathrm{~mm} \mathrm{Hg}$ & $165(113-210)$ & $56(22-90)$ & $188(124-252)$ & .000 \\
\hline Lactato $\mathrm{mmol} / \mathrm{L}$ & $1.37(1.0-1.79)$ & $1.95(1.53-2.37)$ & $1.2(0.86-1.64$ & .000 \\
\hline
\end{tabular}


UTI (VMI)

ALTA DOMICILIO

ANTICOAGULACIÓN

DOMICILIO

TRANSFERENCIA

ANTICOAGULACION

HOSPITALIZACIÓN

\section{Anticoagulación}

La profilaxis tromboembólica con HBPM (Heparinas de Bajo Peso Molecular), es recomendada para todos los pacientes hospitalizados con COVID-19, los estudios encuentran disminución de mortalidad en pacientes que reciben anticoagulación, se reporta un punto de corte de 1500 de Ddímero para aumento de riesgo de eventos tromboembólicos (24), en los pacientes del estudio se optó por uso de HBPM, con valores de D-Dímero desde $1000 \mathrm{ng} / \mathrm{ml}$, debido a mayor riesgo de presentación severa de la enfermedad y muerte, en la población de estudio con este corte, se aplico el uso de anticoagulación en domicilio en los pacientes que fueron dados de alta, pero que mantenía aun valores de D-Dímero $>1000 \mathrm{ng} / \mathrm{ml}$. Se debe considerar que la trombosis en pacientes con COVID-19, se debe a la tormenta de citoquinas, injuria hipóxica, disfunción endotelial, hipercoagulabilidad y aumento de la actividad plaquetaria (25), las dosis utilizadas para anticoagulación fueron las recomendadas por ISTH (Sociedad Internacional de Trombosis y Hemostasia) y que fueron adoptadas por la institución.

\section{Corticoides}

Los estudios de corticoides para neumonía viral y SDRA, mostraban resultados mixtos, sin embargo los resultados del Randomized Evaluation of COVID-19 Therapy trial (RECOVERY), fueron publicados ya cerca de finales de Junio, en el que se randomizó a 2104 pacientes con COVID-19 para recibir $6 \mathrm{mg}$ diarios de dexametasona por 10 días y 4321 para recibir los cuidados usuales, encontró una reducción de la mortalidad ( $R R 0.83$ p=.001), demostrando este beneficio en pacientes con más de 7 días de enfermedad y con hipoxemia (26), de esta manera, se instauró el uso de dexametasona en los pacientes de la sala COVID de la institución, desde mediados de julio, por lo que no se pudo hacer inferencia de este efecto en los primeros pacientes del estudio, que fueron objeto de esta revisión.

\section{Mortalidad}

La mortalidad hospitalaria por COVID-19 esta entre el $15 \%$ al $20 \%$, pero aumenta hasta un $40 \%$ en los pacientes que requieren UTI (27), especialmente en los pacientes con ventilación mecánica invasiva. La mortalidad en este estudio fue de 17,1\% (17 pacientes), tanto hospitalizados con 
enfermedad severa y los críticos que necesitaron soporte ventilatorio, considerando que se hospitalizó aproximadamente 298 pacientes con diagnóstico de infección por SARS-CoV-2, entre los meses de marzo hasta Julio, se puede estimar una mortalidad total de 5.7\%, no se incluyeron pacientes con datos incompletos o con resultado de Hisopado pendiente, la unidad hasta el mes de Agosto, registró un total de 348 pacientes. Las tasas de mortalidad varían según las cohortes, reflejando diferencias en los tiempos de entrega de resultados de las pruebas, identificación de caso, y las diferencias en los resultados en los hospitales con distinto equipamiento (28).

Se observó una asociación significativa entre mortalidad y edad, específicamente con 60 años en adelante $(\mathrm{p}=.00)$, no hubo relación entre enfermedades concomitantes y el aumento de la mortalidad $(p=.96)$, en lo que refiere a las características clínicas y mortalidad, no se vio relación significativa con ningún síntoma; la hipoxemia manifestada por los niveles de saturación (.023) y PaFio2 ( $\mathrm{p}=.00)$ mostraron una relación con significativa con mortalidad, se presentó una asociación con la dificultad para transferencias de pacientes críticos y muerte, debido a la saturación en los centros de referencia $(\mathrm{p}=0.56)$.

En lo que refiere a los hallazgos de laboratorio, los valores de D-dímero, se asociaron significativamente, con mortalidad, se observó aumento de la misma, cuando los valores fueron de $>1000 \mathrm{mg} / \mathrm{dl}$, y si se mantenían en de $1500 \mathrm{mg} / \mathrm{dl}(\mathrm{p}=.011)$, el fibrinogeno y el TP (tiempo de protrombina), $\mathrm{LDH}$, presentaron relación que fue significativa a partir del tercer día en promedio luego del ingreso $(p=.06),(p=.070),(p=.009)$ los linfocitos tuvieron un comportamiento inverso proporcional con mortalidad, una disminución de su conteo por debajo de 1000 se relacionó con muerte, los valores de leucocitos y neutrofilos se asociaron con mortalidad cuando sus valores se elevaban por encima de su nivel normal, y este se mantenía durante la hospitalización $(\mathrm{p}=.001)(\mathrm{p}=$ $.000)$

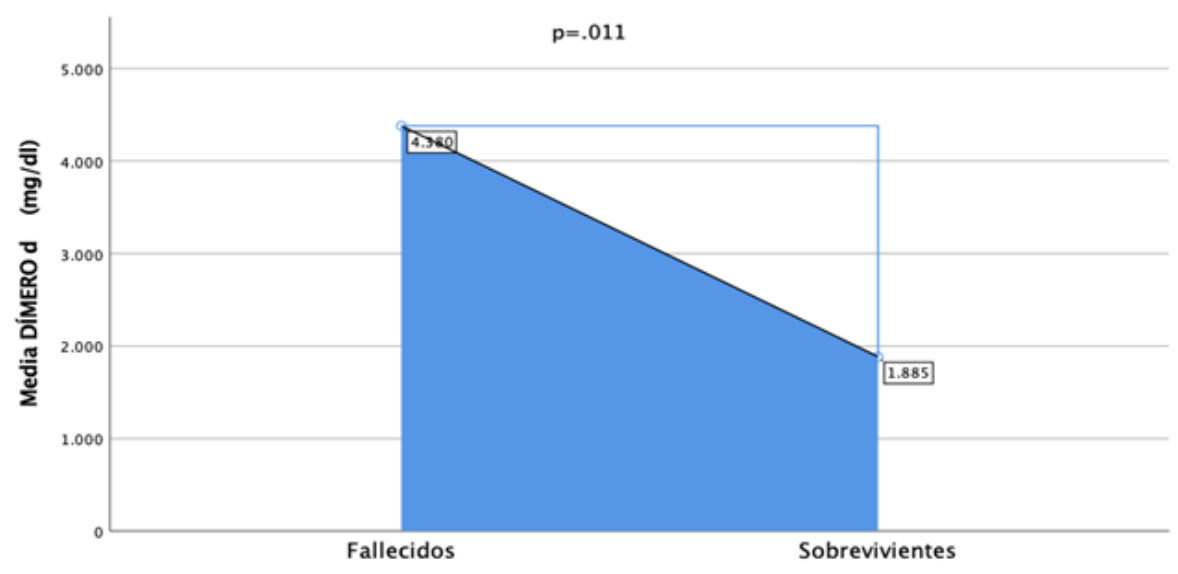




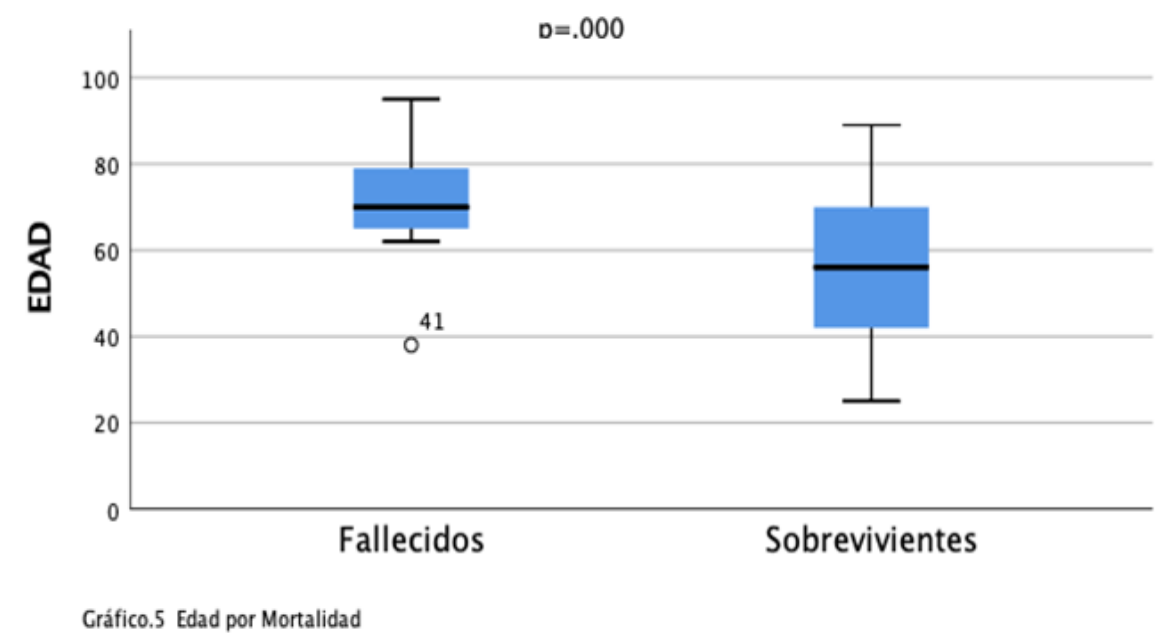

\section{Conclusiones}

En este estudio de los primeros 99 pacientes hospitalizados con diagnóstico confirmado por PCR RT, de Neumonía por coronavirus, en la unidad de COVID de un Hospital de segundo nivel, del Ministerio de Salud Pública, se encontró una edad promedio de 58 años, el 51,5\% (51 pacientes ) fueron mujeres, de estos el 13,1\% (13 pacientes) fueron derivados a Terapia Intensiva o requirieron ventilación mecánica, un total de 55,6\% (55 pacientes) fueron dados de alta al domicilio, con una mortalidad hospitalaria en general de $17.2 \%$. Entre los factores que pueden explicar la mortalidad en esta revisión de serie de casos, esta, el gran incremento de casos; lo cual provoco la saturación de las unidades tanto de hospitalización general y de terapia intensiva en los centros de referencia, en el Hospital Básico, se contó apenas con dos ventiladores y una disponibilidad de 25 camas en la sala COVID, se traduce en que la gran mayoría de los pacientes muertos, se debieron a que no se contó con la respectiva transferencia de pacientes en estado crítico por falta de espacio en la unidad, con indicación para ventilación mecánica, otro factor importante fue la falta de un tratamiento específico contra la enfermedad, que hasta la fecha del estudio no se contaba, la evidencia de los resultados del estudio RECOVERY, acerca del uso de corticoides, aparecerían meses después, contrastando esta cifra de mortalidad con la de los centros a nivel mundial. Se mostraron cifras similares a las que se manifestaron a nivel mundial, en los primeros meses de la pandemia en cuanto a mortalidad hospitalaria se refiere, un estudio publicado entre febrero y abril, del 2020 en Alemania informo una mortalidad entre pacientes hospitalizados por COVID-19, del $22 \%$ esta fue particularmente alta en los pacientes que recibieron ventilación mecánica (53\%) (29)

En el sistema de salud Ecuatoriano, en el que la pandemia de COVID-19 desbordó las capacidades hospitalarias, y considerando las limitaciones de un hospital de segundo nivel, se logró cifras similares a las de otros hospitales a nivel mundial.

\section{Referencias bibliográficas}

1. Huang C, Wang Y, Li X, et al. Clinical features of patients infected with 2019 novel coronavirus in Wuhan, China. Lancet. 2020; 395:497-506. doi:10.1016/S0140-6736(20)30183-5

2. Wang D, Hu B, Hu C, et al. Clinical Characteristics of 138 Hospitalized Patients with 2019 Novel Coronavirus-Infected Pneumonia in Wuhan, China. JAMA - J Am Med Assoc. 2020;323(11):10611069. doi:10.1001/jama.2020.1585

3. WHO-2019-nCoV-clinical-2020.4-eng.

4. Ranieri VM, Rubenfeld GD, Thompson BT, et al. Acute respiratory distress syndrome: The Berlin definition. JAMA - J Am Med Assoc. 2012;307(23):2526-2533. doi:10.1001/jama.2012.566.

5. Li Q, Guan X, Wu P, et al. early transmission dynamics in Wuhan, China, of novel coronavirus- 
infected pneumonia. [published on January 29, 2020]. N Engl J Med. 2020. doi:10. 1056/NEJMoa2001316.

6. Sanche, Steven RESEARCH High Contagiousness and Rapid Spread of Severe Acute Respiratory Syndrome Coronavirus 2, Emerging Infectious Diseases, 2020.

7. Tang N, Bai H, Chen X, Gong J, Li D, Sun Z. Anticoagulant treatment is associated with decreased mortality in severe coronavirus disease 2019 patients with coagulopathy. J Thromb Haemost. 2020;18(5):1094-1099. doi:10.1111/jth.14817

8. Tay MZ, Poh CM, Rénia L, MacAry PA, Ng LFP. The trinity of COVID-19: immunity, inflammation and intervention. Nat Rev Immunol. 2020;20(6):363-374. doi:10.1038/s41577-0200311-8

9. Debuc B, Smadja DM. Is COVID-19 a New Hematologic Disease? 2020. doi:10.1007/s12015020-09987-4

10. Levi M, Thachil J, Iba T, Levy JH. Comment Coagulation abnormalities and thrombosis in patients with COVID-19. 2020. doi:10.1016/S2352-3026(20)30145-9

11. Guan WJ, Ni ZY, Hu Y, et al. Clinical characteristics of coronavirus disease, 2019 in China. N Engl J Med 2020; 382: 1708-20

12. Mehta P, McAuley DF, Brown M, Sanchez E, Tattersall RS, Manson JJ. COVID-19: consider cytokine storm syndromes and immunosuppression. Lancet. 2020;395(10229):1033-1034. doi:10.1016/S0140-6736(20)30628-0

13. McGonagle D, O'Donnell JS, Sharif K, Emery P, Bridgewood C. Immune mechanisms of pulmonary intravascular coagulopathy in COVID-19 pneumonia. Lancet Rheumatol. 2020;2(7):e437-e445. doi:10.1016/S2665-9913(20)30121-1

14. Ten VS, Pinsky DJ. Endothelial response to hypoxia: physiologic adaptation and pathologic dysfunction. Curr Opin Crit Care 2002; 8: 242-50.

15. Yan SF, Mackman N, Kisiel W, Stern DM, Pinsky DJ. Hypoxia/hypoxemia-induced activation of the procoagulant pathways and the pathogenesis of ischemia-associated thrombosis. Arterioscler Thromb Vasc Biol 1999; 19: 2029-35

16. Sandoval Y, Januzzi JL, Jaffe AS. Cardiac Troponin for the Diagnosis and Risk-Stratification of Myocardial Injury in COVID-19: JACC Review Topic of the Week. J Am Coll Cardiol. julio 2020. doi:10.1016/j.jacc.2020.06.068

17. Sandoval Y, Jaffe AS. Type 2 Myocardial Infarction: JACC Review Topic of the Week. J Am Coll Cardiol. 2019;73(14):1846-1860. doi:10.1016/J.JACC.2019.02.018

18. Moore JB, June CH. Cytokine release syndrome in severe COVID-19. Science 2020; 368: 473 474.

19. AlhazzaniW,MøllerMH, ArabiYM,etal. Surviving Sepsis Campaign: guidelines on the management of critically ill adults with coronavirus disease 2019 (COVID-19). Intensive Care Med. 2020;46(5):854-887. doi:10.1007/s00134-020-06022-5

20. Rawson TM, Moore LSP, Zhu N, et al. Bacterial and fungal co-infection in individuals with coronavirus: a rapid review to support COVID-19 antimicrobial prescribing. Clin Infect Dis. Published online May 2, 2020. doi:10.1093/cid/ciaa530

21. Magagnoli J, Narendran S, Pereira F, et al. Outcomes of hydroxychloroquine usage in United States veterans hospitalized with COVID-19. MedRxiv. Preprint posted June 5, 2020. doi:10.1016/j.medj.2020.06.001.

22. Mahévas M, Tran VT, Roumier M, et al. Clinical efficacy of hydroxychloroquine in patients with covid-19 pneumonia who require oxygen: observational comparative study using routine care data. BMJ. 2020;369:m1844. doi:10.1136/bmj.m1844.

23. Rosenberg ES, Dufort EM, Udo T, et al. Association of treatment with hydroxychloroquine or azithromycin with in-hospital mortality in patients with COVID-19 in New York State. JAMA. 2020;323(24):2493-2502. doi:10.1001/jama.2020. 8630.

24. ThachilJ,TangN,GandoS,etal. ISTHinterim guidance on recognition and management of coagulopathy in COVID-19. J Thromb Haemost. 2020;18(5):1023-1026. doi:10.1111/jth.14810.

25. Bilaloglu S, Aphinyanaphongs Y, Jones S, Iturrate E, Hochman J, Berger JS. Thrombosis in Hospitalized Patients with COVID-19 in a New York City Health System. JAMA - J Am Med Asso. 2020. doi:10.1001/jama.2020.13372. 
26. Dexamethasone in Hospitalized Patients with Covid-19 - Preliminary Report. $N$ Engl J Med. julio 2020:NEJMoa2021436. doi:10.1056/NEJMoa2021436

27. Richardson S,HirschJS,NarasimhanM, etal; the Northwell COVID-19 Research Consortium. Presenting characteristics, comorbidities, and outcomes among 5700 patients hospitalized with COVID-19 in the New York City area. JAMA. 2020; 323(20):2052-2059. doi:10.1001/jama.2020.6775.

28. Garg S,Kim L,Whitaker M,etal. Hospitalization rates and characteristics of patients hospitalized with laboratory-confirmed coronavirusdisease 2019-COVID-NET, 14 States, March 1-30, 2020. MMWR Morb Mortal Wkly Rep. 2020;69(15):458- 464. doi:10.15585/mmwr.mm6915e3.

29. Karagiannidis C, Mostert C, Case characteristics, resources use, and outcomes of 10021patients with COVID-19 admitted to 920 observational study, the lancet Respiratory Mdedicine. 2020 vol.8(9)pp.853-862. 